\title{
Pensamiento crítico el reto de la formación filosófica en la actualidad. Un aporte desde el observatorio filosófico virtual.
}

\section{Critical thinking the challenge of philosophical training today. A contribution from the virtual philosophical observatory.}

DOI: $10.32870 /$ sincronia.axxiv.n77.1a20

\author{
Paula Andrea Mora Pedreros ${ }^{1}$ \\ Corporación Universitaria Autónoma del Cauca (COLOMBIA) \\ CE: paula.mora.p@uniautonoma.edu.co ID ORCID: 0000-0001-8070-9885 \\ Dénix Alberto Rodríguez Torres ${ }^{2}$ \\ Universidad Santo Tomás (COLOMBIA) \\ CE: denix.rodriguez@ustabuca.edu.co ID ORCID: 0000-0002-9259-4971 \\ Manuel José Santiago Pajajoy 3 \\ Corporación Universitaria Autónoma del Cauca (COLOMBIA) \\ CE: manuel.santiago.p@uniautonoma.edu.co ID ORCID: 0000-0003-1730-9218
}

\footnotetext{
${ }^{1}$ Corporación Universitaria Autónoma del Cauca. Licenciada en Filosofía y Letras y Magister en Etnoliteratura, Universidad de Nariño; Especialista en Pedagogía del Aprendizaje Autónomo y Especialista en Educación Superior, UNAD; Magister en Tecnología Educativa con énfasis en innovación educativa, Tecnológico de Monterrey; Candidata a Doctor en Filosofía, Universidad Pontificia Bolivariana. Certificada como tutor virtual, OEA-OEl; director virtual, diseñador de material didáctico, UNAD; en didácticas digitales, Universidad de Catamarca; Directora del grupo Amautas Categorizado en B en Colciencias y participante Grupo GIICSH, pasante internacional en Israel y Chile en proyectos de argumentación; docente Investigadora de la Corporación Autónoma del Cauca. Email: paula.mora.p@uniautonoma.edu.co

${ }^{2}$ Universidad Santo Tomás. Licenciado en Filosofía. Magíster en Educación. Doctor en Educación. Docente Investigador. Posdoctorado en Epistemología. Par Investigador Colciencias. Docente Universitario. Editor revista TEMAS. Universidad Santo Tomás. Docente Invitado a la Maestría en Pedagogía por la Universidad Industrial de Santander. UIS. Con el seminario: El Saber Pedagógico: Epistemología e Historia de la Pedagogía. Docente Invitado a la Maestría en Ciencias y Tecnologías Ambientales, por la Universidad santo Tomás, con el seminario: Epistemología Ambiental. Escritor y coautor de diferentes libros, capítulos de libro y diversos artículos científicos. Email: denix.rodriguez@ustabuca.edu.co

${ }^{3}$ Abogado, Especialista en Penal, Maestrante en Propiedad intelectual de Flacso- Argentina. Docente Investigador Grupo GICSH. Corporación Universitaria Autónoma del Cauca, Colombia, Manuel.santiago.p@uniautonoma.edu.co
} 
Recibido: 01/04/2019

Revisado: $31 / 10 / 2019$

Aprobado: 15/11/2019

\section{RESUMEN}

Todo proceso educativo ha de empezar en la niñez y, por lo tanto, la importancia de incluir la filosofía en el currículo, aún desde la primera infancia; es aquí donde la investigación tiene relevancia al evidenciar el proceso formativo de los docentes que adoptan no sólo metodologías como la comunidad de indagación propia del programa FpN propuesta por Lipman (1969), sino además la exploración de nuevos híbridos que permiten evidenciar propuestas alternativas en la intención docente de suscitar pensamiento y reflexión en el quehacer pedagógico, en la búsqueda de un proyecto educativo que tiene como objetivo enseñar a pensar, a sentir y a vivir de un modo riguroso, crítico, creativo, solidario y cuidadoso (Miranda Alonso, 1995, citado por Espinosa, 2007).

Es por lo que, la misión de buscar alternativas para consolidar proyectos educativos tejidos con el contexto y la realidad del niño y del maestro, de forma tal que la Filosofía se convierte en una herramienta didáctica, reflexiva y transformadora para lograr cambio de pensamiento y acción crítica en cada niño que tiene vívido su asombro y curiosidad.

Finalmente este artículo surge en el marco de los proyectos de investigación que acompaña el Grupo Amautas, el grupo GIISCH y el Observatorio Filosófico Virtual, con la firme creencia que exponían Lipman \& Sharp (1990), considerando que la FpN es un buen camino para que surja el diálogo reflexivo y comunicativo, tanto por los temas que plantea, como por el modo en que se hace, con ello lograr una mayor comprensión de las vivencias de manera autónoma, crítica y creativa en beneficio del ser individual y colectivo de la sociedad

Palabras clave: Filosofía para niños. Didáctica de la filosofía. Comunidad de indagación. Pensamiento crítico.

\section{ABSTRACT}

All educational processes must begin in childhood and, therefore, the importance of including philosophy in the curriculum, even from early childhood; It is here that research is relevant to highlighting the educational process of teachers who adopt not only methodologies such as the research community of the FpN program proposed by Lipman (1969), but also the exploration of new hybrids that show alternative proposals in the teaching intention to arouse thought and reflection in the pedagogical task, in the search of an educational project that has the objective of teaching to 
think, to feel and to live in a rigorous, critical, creative, solidary and careful way (Miranda Alonso, 1995, cited by Espinosa, 2007).

That is why, the mission of finding alternatives to consolidate educational projects woven with the context and the reality of the child and the teacher, in such a way that Philosophy becomes a didactic, reflective and transformative tool to achieve change of thought and action criticism in every child who has vivid his amazement and curiosity.

Finally, this article arises within the framework of the research projects that the Amautas Group and GIISCH grupo and the Virtual Philosophical Observatory accompany, with the firm belief that Lipman \& Sharp (1990) expounded, considering that the FpN is a good way for reflexive dialogue to emerge and communicative, both for the issues raised, and the way it is done, thereby achieving a better understanding of the experiences in an autonomous, critical and creative way for the benefit of the individual and collective being of society.

Keywords: Philosophy for children. Philosophy didactics. Community of inquiry. Critical thinking.

\section{Aproximación histórica}

Lo aquí expuesto puede derivar en muchas aristas y discusiones que dependen de los contextos socioculturales donde nos situemos. Podríamos disertar sobre los procesos educativos que han antecedido la realidad educativa de nuestros niños y niñas a lo largo de la historia. Para el caso colombiano, existen diversas apuestas orientadas a la generación de competencias e iniciativas que potencian el pensamiento crítico, pero en realidad no es posible no generalizar ni identificar un horizonte común, aún más cuando existen hondos vacíos conceptuales que pudieran otorgar un asidero a quienes desde las aulas $u$ otras ventanas quisieran potenciar verdaderamente un pensamiento crítico.

Sobre dicha categoría -Pensamiento Crítico- no existe claridad sobre su génesis. Hay quienes dicen que el pensamiento crítico tiene conexión directa con un libro escrito en 1952 por Max Black, titulado El pensamiento crítico. En dicho libro se procura acercar a los estudiantes a la lógica del pensamiento. Otros aluden su relación con la lógica británica, Susan Stebbing, que desde su obra Thinking to Some Purpose, pretendió demostrar el valor del pensamiento lógico. Podemos seguir una línea delgada a modo de estado de arte que nos permitiría rastrear aún más agudamente la evolución e instauración del pensamiento crítico, no por ello podemos dejar de aludir a Dewey, 
quien enfatiza su interés por la educación a finales del siglo XIX. La educación situada en esta época apuesta de manera decidida por la ciencia en el currículum, apartando los clásicos. Para Dewey ello significaba el triunfo de los flexibles y dinámicos métodos de investigación de la ciencia frente a la inflexibilidad y estatismo del humanismo clásico.

Dewey siempre soñó con que los estudiantes estuvieran realmente comprometidos con la investigación científica, ello realmente permitiría hablar de una sociedad civilizada, preparada para abordar los problemas. En su importante obra, aparecida una década después: Democracia y Educación, el pedagogo mantiene su idea de la investigación científica como modelo para la educación, pero enfatizando en la importancia educativa del pensamiento. Allí expresaba Dewey la importancia de aprender cómo enseñar a los niños y niñas a que piensen por sí mismos si se desea tener una democracia valiosa. Es tan importante un sujeto pensante como una sociedad investigadora. De ahí que Giroux (2003) Propone un docente que acompaña este ejercicio desde la perspectiva más fenomenológica y neormarxista del pensamiento y la práctica educativa, es decir que impacten sobre la forma en que los docentes orientan los currículos y consideran las dimensiones éticas de formación del ciudadano como ente pensante y actuante.

\section{Desarrollo de Habilidades para el Pensamiento Crítico}

Por el año 1963, Robert Ennis publicó en Harvard Educational Review, una definición de pensamiento crítico. Un artículo orientado a ampliar la influencia del concepto por una educación para el pensamiento. De todas formas, debemos a Dewey un acertado direccionamiento al papel de la filosofía frente al pensamiento crítico. Para Dewey la filosofía es una forma especial de cognición no científica que se interesa por el juicio de valor como única forma de investigación, el juicio del juicio, -un criticismo del criticismo-. Por ello resulta fundamental releer el capítulo final de la obra fundamental sobre metafísica de Dewey: Experience and Nature (1929) que expresa de manera clara la conexión entre filosofía y pensamiento crítico.

Como ya se ha anotado, la idea de generar habilidades para el pensamiento crítico ha desarrollado en las últimas décadas toda una desiderata de ideas, investigaciones, proyectos e 
iniciativas loables en educarlo en los estudiantes, aunque dada su vaguedad conceptual ha llegado a satanizar o tergiversar en seudopensamiento crítico, censurado en el peor de los casos en quienes en sus discursos y currículas dicen incentivarlo.

Ronald Barnett (2001) en su sonado libro Los límites de la competencia, alude al pensamiento crítico como parte de la lista del vocabulario perdido en la universidad y por ende en la educación en general, ya que los sistemas capitalistas que permean directamente la educación y encuentran resistencia en quienes piensen de una manera más profunda y reflexiva. Igualmente sucede en la bella obra de Martha Nussbaum: Sin fines de lucro, por qué la Democracia necesita de las humanidades (2010). Vale la pena citar un apartado del artículo Del modelo de desarrollo económico al paradigma de desarrollo humano (2012) de la Revista Complutense de Educación:

La "crisis silenciosa" generada por las naciones "sedientas de ingresos", desechan otra aptitud en la formación de las personas: El Pensamiento Crítico, aptitud abordada de manera recurrente en Nussbaum. Se referencia inicialmente como un elemento desaconsejado, aludiendo al poco interés que una educación para el desarrollo económico pone en su cultivo, ya que puede convertirse en una amenaza -así sea de pequeñas élites- si éstas reflexionan relatos sobre la historia social o datos económicos, de donde podrían surgir mayores disertaciones en torno a cuestiones de género, raza o sobre las "bondades" de las inversiones extranjeras para los sectores de la población rural o para la supervivencia de la democracia, donde son perceptibles las desigualdades de oportunidades básicas. (Argüello, Cabeza, Cardona, Hernández y Rodríguez, 2012, p. 408).

El pensamiento crítico no sería un componente importante de la educación para el crecimiento económico, y en efecto, no lo ha sido para los estados que persiguen esa meta sin descanso como Guajarat, al oeste de la india, conocido por su combinación de alta complejidad tecnológica con docilidad y pensamiento grupal o grouptihink (p .42).

Por tanto, la libertad de pensamiento en el estudiante se torna peligrosa, debido a que las pretensiones están orientadas a obtener personas o trabajadores obedientes, pero altamente productivos, donde el pensamiento crítico será "desalentado" para dar prioridad al trabajo y al 
rendimiento económico. Comprender la historia, con todos sus horrores y desaciertos, produciría una reflexión crítica del presente, nada conveniente para los intereses económicos de las naciones, que reducirían su historia a los triunfos obtenidos y a las metas globales, ocultando las penosas escenas de injusticias de clase, casta, género, etnia o religión (Argüello, et. al., 2012).

La apuesta por el desarrollo de iniciativas en el aula orientadas a estimular la creatividad y el pensamiento crítico de los niños y niñas compromete de manera considerable al maestro o tutor. Si este no posee la iniciativa, pero además las herramientas conceptuales y actitudinales para hacerlo, podemos decir que es una iniciativa que nace muerta. (Amador; Vélez; Cardozo; Pinto y Mora, 2017).

Por lo anterior, en el desarrollo de la propuesta formativa se ha buscado potenciar en los infantes la curiosidad y el maravillarse que les viene dado en medio de la cultura de lo obvio a la cual asistimos y que nos ha imbuido silenciosamente la cultura capitalista y la obsolescencia no sólo tecnológica sino vital. Estimular la admiración por lo sencillo e irrelevante no es cuestión fácil, pero tampoco imposible. El pensamiento crítico y filosófico en los infantes permite el desarrollo de la sensibilidad al contexto. En este sentido estimular el pensamiento frente a la toma de postura a circunstancias excepcionales o irregulares (acciones bélicas, violentas, injusticias, etc.) alientan al pensamiento crítico en su primera forma. El análisis de situaciones sociopolíticas regionales, permiten a los niños el análisis, la argumentación y toma de postura, aunque parezca irrisorio. (Alvear y Mora, 2011). De forma específica se presenta la gráfica compilada por Paul, R., \& Elder, L. (2003) que permite establecer una ruta de los elementos del pensamiento y se articula a las propuestas didácticas para su desarrollo. 


\section{Gráfica 1 Elementos del pensamiento}

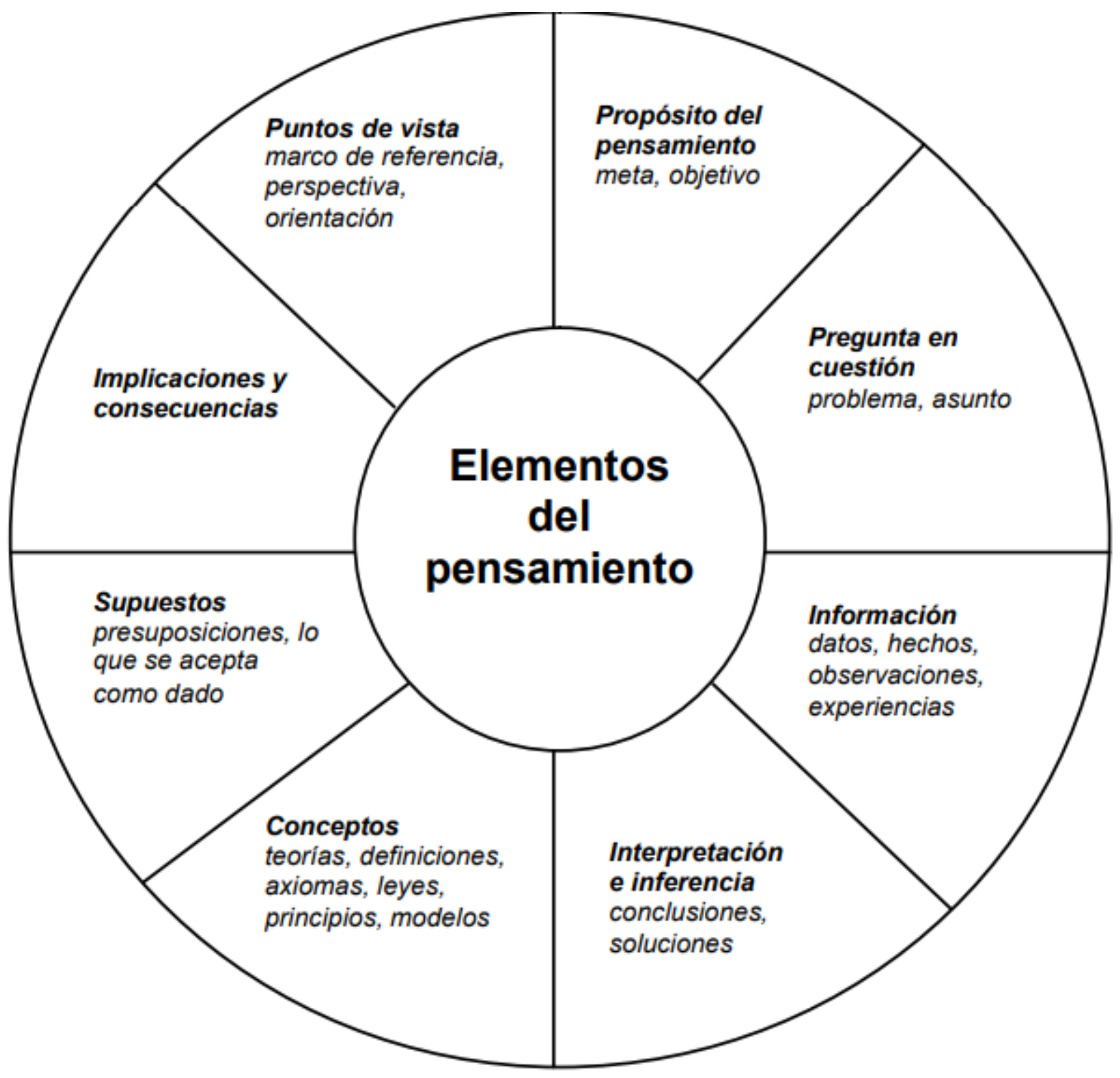

Fuente: Paul y Elder, 2003, p. 5

El niño puede desarrollar configuraciones globales, por ejemplo, sobre el medio ambiente y el impacto de la mano del hombre sobre la naturaleza, además de ir configurando conciencia ambiental, genera reflexiones de diferente orden geoespacial según el caso analizado. Un elemento clave orientado a la generación de pensamiento crítico, reclama evidencias verificables, no admite 
generalizaciones ni juicios a priori. Es indiscutible que el niño piense no sobre supuestos si no basado en estadísticas, en cifras, en realidades concretas constatables para él.

Finalmente, la capacidad de situar culturalmente el pensamiento y contextualizarlo, permite una valoración de acciones procedentes y aceptadas éticamente en algunos lugares y en otros no, ello permite desarrollar capacidad para el diálogo, el multiculturalismo, la tolerancia y la imaginación narrativa como la posibilidad de ponerse en el lugar del otro -en palabras de Nussbaum (2010)- y clave de la educación progresista.

\section{Desarrollo del Pensamiento Crítico y Filosófico en Niños}

En consecuencia, de lo anterior puede expresarse que existen diversas formas en las que derivan el pensamiento con criterios y la sensibilidad al contexto y que bien pudiéramos pensar en el trabajo del pensamiento filosófico con el niño en el desarrollo de una comunidad de indagación. Por ejemplo, se planteó una serie de situaciones ficticias y lo que se desea potencial o cultivar es la imparcialidad, que es finalmente un criterio más extenso de justicia. Y una forma de potenciar y asumir esa imparcialidad es por ejemplo esperar el turno. Este ejercicio es tomado del libro Asombrándose ante el mundo. Manual del profesor para acompañar a Kio y Gus (1993), del programa de Filosofía para Niños entre nueve y diez años.

Cuando los niños realizan este ejercicio aplican el criterio de esperar el turno (esto es, la reciprocidad, la imparcialidad o la justicia) hacia o frente a una media docena de situaciones específicas que exigen la sensibilidad al contexto. Mediante la discusión de grupo se vieron capacitados para distinguir entre aquellas situaciones en las que es apropiado posicionarse la espera del turno y aquellas en las que es dudoso. Cuando ejercicios de este tipo se utilizan en una comunidad de investigación (indagación), estamos asegurando el desarrollo del pensamiento crítico en el aula. Es evidente que no es la única forma de lograrlo, pero resulta ser una importante.

Por su puesto que estas importantes reflexiones no pueden desligarse de la realidad global a la cual asistimos y que replantea de hecho la manera de educar, de enseñar y de pensar el mundo. Una cuestión inaplazable para el debate a todas las esferas es sin duda la educación, siendo esta la 
preocupación de intelectuales, investigadores, universidades y muchos Estados. (Barreda; González; Constain y Mora, 2019).

Hace apenas unos pocos años, en 2005, el gran sociólogo y filósofo polaco Zygmunt Bauman, escribía un libro muy liviano en extensión y profundo en contenido titulado: Los retos de la educación en la modernidad líquida (2005), que sintetiza de manera lúcida la condición de los individuos en la sociedad de consumo del siglo XXI, donde magistralmente se delimitan los contornos de un estado de cosas en el que los individuos convertidos en consumidores han perdido contacto con todas las referencias ideológicas, sociales y de comportamiento que habían determinado su actuación en siglos anteriores. Ya Giroux (2003) consideraba la importancia del "ideal dialógico" como una oportunidad de sentido formativo que permite la construcción de una sociedad, en términos de Bauman.

Hoy la vida se acelera, por la necesidad, casi obligación de aprovechar cientos de oportunidades de felicidad como sean posibles, cosa que nos permite ser alguien nuevo a cada momento. Por ejemplo, la identidad se construye por medio de accesorios comprados que aparecen en el mercado en número que se multiplica hasta el infinito y llega a ser incontrolable, igual que el cúmulo de información con el que nuestro criterio es bombardeado desde todos los flancos. Lo anterior incide fuertemente en la manera como nos relacionamos con el saber, el trabajo y la vida en general. La educación entonces, en la era de la modernidad líquida, ha abandonado la noción de conocimiento de la verdad útil para toda la vida y se ha sustituido por la de conocimiento de usar y tirar, válido mientras no se diga lo contrario.

Estas ideas iluminadoras conectan de manera importante con nuestra reflexión y propósito de pensar una formación filosófica en infantes pero desde el desarrollo de habilidades para el pensamiento crítico, la creatividad y el asombro como categorías socavadas en la modernidad acelerada y líquida (Bauman), ya que todo está dado, todo está preparado y listo, ahorrando la angustia del pensar y el decidir que para muchos resulta fatigosa como lo evoca Estanislao Zuleta en su Elogio de la dificultad (1980). 
Uno de los asuntos sin duda alguna abordados por Bauman (2005) es el del conocimiento, que reflejaba en el campo educativo algo que tenía su puesto dado, ya que duraba, así como la educación misma que ofrecía un conocimiento para atesorarse, para siempre. En el contexto de la modernidad líquida esta idea ha perdido su encanto. Hoy, se encuentra en tela de juicio lo invariable de la idea. "las características constitutivas de la educación que hasta ahora habían soportado todos los retos del pasado y habían emergido ilesas de todas las crisis“" (Bauman, 2005, p.75).

Hoy, todo es relativo, dinámico y este devenir les hace obsoleto TODO. No podemos presentarnos en público por ejemplo con un móvil de modelo pasado. Nuestro mundo recuerda cada vez más la Ciudad Invisible de Leonia de Ítalo Calvino donde "la opulencia puede medirse no tanto por las cosas que se fabrican, se compran y se venden cada día...sino, antes bien, por las cosas que se tiran diariamente para dejar lugar a las nuevas“" (Bauman, p. 29). Ante semejante situación es recurrente la pregunta por el qué y cómo enseñar y formar "para un mundo en devenir", que implica ver el conocimiento como mercancía que se compra y se patenta así sea por porciones.

Formar para la vida y pensar que ese conocimiento nadie nos lo podrá quitar es una promesa que ha caído en desuso. ¿Qué enseñar entonces a nuestros niños y niñas en la era de la incertidumbre?, es más cómo formar para la incertidumbre podríamos preguntarnos con Morín, como bien lo plantea en Los siete saberes necesarios para la educación del futuro (2001). En medio de este panorama sombrío y desesperanzador, como náufragos al límite buscamos resquicios para asir nuestra determinación, faros iluminadores que enfoquen nuestro horizonte y den sentido a nuestra labor educativa y nos pongan a buen recaudo mientras la vertiginosidad del tiempo nos lance a sus derivas nuevamente.

Con todo esto, esta iniciativa referencia a Lipman (1991), quien propugnó por la aparición de un pensamiento orientado hacia la excelencia, de un pensamiento complejo que comprendiese no sólo un pensamiento crítico, sino también creativo. Creó el programa de filosofía para niños con el fin de desarrollar sus habilidades del acto de pensar. Lipman definió el pensamiento crítico como 
“un pensamiento que 1) facilite el juicio al 2) confiar en el criterio, 3)sea autocorrectivo y 4) sea sensible al contexto“ (Lipman, 1995. Trad. Thinking in Education).

Vale la pena estudiar detenidamente su propuesta desglosada en los cuatro criterios a saber:

1. Los juicios son resultado del pensamiento crítico. 2. El pensamiento crítico se apoya en criterios. 3. El pensamiento crítico es autocorrectivo y 4. El pensamiento crítico es sensible al contexto.

Lipman, a su vez cree que el enfoque de la comunidad de investigación es la pedagogía que se adapta mejor a la tarea de reforzar el razonamiento y el juicio, sea que esta investigación sea científica o no: los niños se escuchan entre sí, cuestionan el tema a partir de las ideas de los demás, ponen en duda las opiniones que no se basan en razones válidas, se ayudan entre sí para sacar las inferencias de su discusión y buscan reconocer las suposiciones de cada uno. Para Lipman (1995), “lo que caracteriza a la comunidad de investigación (indagación) es el diálogo guiado por la lógica. Para saber qué hacer, hay que razonar (p.281).

La institución social del jurado representa según él, un modelo de diálogo deliberativo en el que se manifiesta el pensamiento crítico: los miembros de este selecto colectivo reflexionan juntos y corrigen mutuamente sus errores de razonamiento (procedimiento autocorrectivo) sin perder de vista las particularidades de la situación que debaten (sensibilidad ante el contexto) para obtener juntos un veredicto (juicio basado en criterios). De la misma forma que se otorga confianza a las deliberaciones de un jurado guiado por jueces competentes, también podemos otorgar confianza a las discusiones de las comunidades de investigación guiadas por docentes calificados. Lipman concluye como sigue: “ ¿Qué mejor forma de preparar a los alumnos para la vida que hacerlos capaces de participar en comunidades deliberativas que traten los problemas que les aquejan? " (Boisvert, 2004, p. 286).

El agenciamiento de las comunidades de indagación en nuestros contextos escolares y en su gran mayoría en planteles educativos latinoamericanos, plantea desde luego el des-aprendizaje de conductas, formas de ser, de enseñar y de aprender, establece todo un desafío ante la homogeneización del saber y el adoctrinamiento del cual padecen aún cientos de planteles 
educativos, anclados en modelos pedagógicos bancarios (Freire, 2002), donde el transmisionismo de información es lo central y la estandarización del "conocimiento" concentra todo valor.

Desde los mismos métodos de enseñanza tradicionales y disposiciones geográficas de las aulas de clase deberían empezar a revisarse. Un aula de clase cuadriculada, donde todos miran al frente, pero se dan la espalda es ya una barrera para emprender un cambio paradigmático en la enseñanza.

\section{Pensamiento Pedagógico Crítico}

En este recorrido de reflexión y análisis del pensamiento crítico en el sistema educativo, es oportuno asomarse al Movimiento por la Escuela Nueva, quien hizo la crítica de los métodos tradicionales de la educación. El marxismo y el positivismo también lo hicieron a su modo. Desde otra ventana igualmente los existencialistas y fenomenologístas, lo abordaron como pensamiento antiautoritario. Todo ello ocurría mientras se interrogaban qué era lo que hacía al hombre que se odiara tanto inspirados en las dos guerras mundiales.

Desde la anterior perspectiva, la crítica a la educación se hizo radical, entonces surgieron pensadores como Louis Althusser (1969) quien hablaría de los aparatos ideológicos del Estado. Por otro lado, los sociólogos franceses Pierre Bourdieu y Jean Claude Passeron (1970) lo hicieron desde la Reproducción. Claude baudelot y Roger Establet (1971) hablaron de la Escuela Capitalista en Francia. Todas estas posturas tuvieron gran acogida e interpelaron demostrando cómo la escuela reproduce la sociedad, de aquí que se les conozca mo crítico-reproductivistas.

En una suerte de acercamiento a los autores mencionados, es posible indicar que Althusser sostuvo que la misión propia de la escuela capitalista consistiría en la reproducción de la sociedad y que toda acción pedagógica sería entonces una imposición arbitraria de la cultura de las clases dominantes. Por su parte Bourdieu y Passeron, argumentaron que la escuela se constituía en el instrumento más acabado del capitalismo para reproducir las relaciones de reproducción y la ideología del sistema. baudelot y Establet, analizando la escuela capitalista en Francia, demostraron 
la existencia de dos grandes redes escolares, que corresponderían a las clases fundamentales de la sociedad: la Burguesía y el Proletariado.

Desde estos presupuestos se vislumbra la visión crítica de la escuela y la imperiosa necesidad del pensamiento crítico en la concepción de la misma y como baluarte de desarrollo en las aulas y en los mismos sistemas educativos. Acota Jesús Palacios, educador español en su obra la Cuestión Escolar, después de un análisis de las teorías "reproductivistas" en las cuales fundamenta su análisis, que:

[...] la escuela no es ni la causa, ni el instrumento de la división de la sociedad en clases; es su consecuencia. Alteraciones en los métodos y en las técnicas escolares o en la democratización de la enseñanza no alteran ese cuadro. (Gadotti, 2008, p. 202).

El pensamiento crítico y antiautoritario que es posible encontrar en la llamada Escuela de Frankfurt (Alemania) constituye uno de los referentes más importante, donde se inspira el pedagogo contemporáneo Henry Giroux, quien partiendo de la teoría crítica de la sociedad, elaborada por la Escuela de Frankfurt, hace la crítica del pensamiento crítico, poniendo en evidencia sus limitaciones. Para Giroux en sus teorías del pensamiento crítico no hay lugar para el conflicto y la contradicción, ya que (según Giroux) tanto Bourdieu como Bernstein, presentaban una versión de dominación en la cual el ciclo de la reproducción parece inquebrantable. Aunque estos dos autores proporcionen análisis clarificadores sobre la relativa autonomía de las escuelas y la naturaleza política de la cultura como fuerza reproductora, terminarían ignorando o minimizando las nociones de resistencia y lucha contrahegemónica.

Rodríguez, Gutiérrez, García, Martínez y Ricón (2019), en su artículo “Correlación de los modelos pedagógicos y el currículo en el contexto educativo" publicado en ese año en la revista de humanidades Temas, hacen un abordaje importante sobre los modelos pedagógicos más representativos y su relación con el currículo en el contexto educativo. Llama la atención este acercamiento a modelos pedagógicos como: el tradicional, conductista, progresista, cognoscitivista y el crítico-radical; que, aunque tengan teorías distintas sobre el desarrollo del aprendizaje, 
permiten tener una aproximación cercana al modelo de educación que se ajusta a las necesidades de cada contexto e individuo. Vale la pena resaltar, el último modelo allí destacado: El modelo pedagógico crítico-radical, "que por su parte, se enfoca en el aprendizaje de habilidades criticoreflexivas para permitir un cambio social, propone una relación entre maestro-alumno para promover los procesos de concientización y reflexión". (Flórez, 1994, citado en Rodríguez, et. al. 2019, p. 145). En la paleta de posibilidades y propuestas lógicamente se sitúa este modelo críticoradical, que constituye una apuesta más enfocada en las habilidades y capacidades de maestros y estudiantes enfocadas a la concientización y reflexión de la realidad. No obstante, mientras las escuelas sigan los modelos hegemónicos, neoliberales y capitalistas de la educación, todo ello no serán más que nobles intentos pero que finalmente quedan vacíos ante las intencionalidades de los Estados y su inquebrantable afectación en la educación. En consecuencia, los autores concluyen que: "Los modelos pedagógicos parten de concepciones diferentes del ser humano y del tipo de hombre y de sociedad que se quiere contribuir a formar, donde todo modelo pedagógico se debe llevar de manera pedagógica según su realidad, permitiendo que los alumnos adquieran un aprendizaje productivo, integral para ser capaces de construir sus propios significados. (p. 152).

\section{Desafíos desde el aula}

Según Jacques Derrida (2002) en su discurso magistral La Universidad Sin Condición, (universidad moderna) las aulas de clase son los espacios privilegiados para que nada absolutamente nada quede de pie ante la crítica, nada debe estar resguardado al cuestionamiento. En este sentido, las aulas y los grupos de discusión e investigación han de ser críticos por excelencia, aunque como ya se ha anotado no resulta fácil formar para el pensamiento crítico, pero es el desafío. Es imperativo formar para llegar a acuerdo no solamente desde los consensos sino desde los disensos mismos, aprendiendo entonces así a discutir sin agredirnos, a sentar postura y argumentar sin necesidad del insulto, la agresión verbal o física, sino desde el marco del respeto y la tolerancia. Para alcanzarlo se debe surtir entonces un largo proceso de decolonización de ideas fijas y egoístas que por años han marcado nuestras culturas y pueblos. Desaprender la violencia para dar el paso al diálogo es toda 
una tarea inconclusa en muchos de nuestros contextos socioculturales que de manera ancestral han vivido la violencia armada, física y sicológica, han padecido la usurpación histórica de sus ideas y bienes. El machismo ancestral que ha perpetuado brechas copiosas de género durante años, no permiten por ejemplo el reconocimiento igualitario de capacidades entre hombres y mujeres, aunque en la segunda década del siglo XXI, se hayan alcanzado ciertos peldaños por la equidad de género.

La comprensión de una ética planetaria (Morín) que nos recuerda que todas y todos somos ciudadanos del mundo en esta tierra-patria, constituyen haz de luz hacia una ética del género humano. Enseñar el respeto por las ideas de los demás, aunque disienta de ellas, es un baluarte con el que hay que educar a los niños y las comunidades de discusión y aprendizaje son espacios para hacerlo. Sin reservas, nuestros planteles educativos latinoamericanos están invitados a potenciar en los niños la capacidad de asombro ante un mundo robotizado, automatizado y enajenado totalmente a la tecnología. El transhumanismo, ha legado a la máquina las emociones humanas, los trabajos intelectuales y el placer de amar, soñar, crear y recrear la vida. Nuestro proyecto de vida ahora tiene nombre propio: Software, incrustado en un artefacto con figura humana, que lo hace todo, clamando a cambio más energía que amor.

Pensar el desarrollo de habilidades para el pensamiento crítico en las esferas educativas con presencialidad o virtualidad, implica a su vez otra emergencia educativa inaplazable: la formación ciudadana. No resulta lejana esta necesidad en la sociedad y menos extraña para teóricos como Adela Cortina, Chantal Mouffe, Edgar Morín, Martha Nussbaum, Guillermo Hoyos, Enrique Chaux, entre otros contemporáneos que se interrogan por el papel de la educación y de las humanidades en la formación ciudadana de los niños y jóvenes. No puede ignorarse que la sociedad, espera de la educación, la generación de personas con sentido social y capacidad crítica en conjunto con las capacidades cognitivas desarrolladas o adquiridas a lo largo de su formación escolar. Se guarda entonces la esperanza por parte de la sociedad la generación de hombres y mujeres que sean ciudadanos responsables y comprometidos con los contextos, con la realidad local y planetaria que les ha correspondido vivir. En el libro: "Influencia de las humanidades en la construcción holística de 
la ciudadanía universal desde Martha Nussbaum" (2019), Dénix Rodríguez, plantea la siguiente pregunta: ¿Es tarea de la Universidad la formación del pensamiento crítico? A este interrogante planteado a estudiantes universitarios y docentes de dicho nivel, se halla una clara división, entre quienes otorgan a las universidades exclusividad en la formación de pensamiento crítico y entre quienes creen que operarían otras esferas. A lo que el autor a agrega:

Es factible pensar otros escenarios para el desarrollo del pensamiento crítico, entiéndase por sociedad, movimiento político o ciudadano, agremiación no educativa, entre otras. No obstante, las humanidades forjan habilidades para el pensamiento crítico, por medio del debate, la disertación, eventos de orden académico y muchos espacios que sólo en la rigurosidad de la academia (escuela) pueden fundamentarse. No se descarta la presencia de otros espacios. Nussbaum al respecto agrega que en la universidad se debe forjar el pensamiento crítico y es a esta posibilidad la que ataca o socava directamente el paradigma de desarrollo económico presente en nuestras democracias. (Rodríguez, 2019, p. 130).

Es claro que aunque existen diversos escenarios donde podría formarse o fundamentarse el pensamiento crítico en la esfera de los diferentes escenarios que ofrece la sociedad y la cultura misma, en la academia se hallan las condiciones favorables para desarrollar y potenciar las habilidades para el pensamiento crítico, de ahí la importancia de proveer espacios y didácticas, estrategias y herramientas que apunten a este propósito que trasciende sin duda alguna los modelos pedagógicos centrados en el transmisionismo y la repetición asentida de la información allí transmitida pero lejos del rasero del análisis, la crítica y la reflexividad.

Urge que nuestras niñas y niños desarrollen y discutan nuestro papel por ejemplo en el planeta, desde los cientos de casos locales y globales que sin permiso han tocado nuestros contextos, y ello traducido en sequías, hambrunas, enfermedades virales, cambios climáticos insospechados, extinción sistemática de especies, y se piense en medio de este maremágnum de acontecimientos la viabilidad de la especie humana. Cómo dejar mejores personas para el planeta y no un mejor planeta para las personas tal vez sea la cuestión. 
Pensar el asunto del calentamiento global en la esfera de la filosofía para niños tal vez no disguste a los sapientes filósofos que cuestionan el proyecto de Lipman, y si permita dar el paso a una epistemología ambiental como bien lo propone el ilustre Enrique Leff (2006).

La crisis ambiental es, sobre todo, un problema del conocimiento, que lleva a repensar el ser del mundo complejo, a entender sus vías de complejización (la diferencia y el enlazamiento entre la complejidad del ser y el pensamiento), para desde allí abrir nuevas vías del saber en el sentido de la reconstrucción y la reapropiación del mundo. La racionalidad dominante del sistema mundo hegemónico descubre la complejidad cuando se confronta con sus límites. La complejidad ambiental irrumpe desde su negación por el pensamiento metafísico y científico, desde la alienación y la incertidumbre del mundo economizado, arrastrado por una racionalidad insustentable y por un proceso incontrolable de producción hacia la muerte entrópica del planeta.

La crisis ambiental es, sobre todo, un problema del conocimiento, que lleva a repensar el ser del mundo complejo, a entender sus vías de complejización (la diferencia y el enlazamiento entre la complejidad del ser y el pensamiento), para desde allí abrir nuevas vías del saber en el sentido de la reconstrucción y la reapropiación del mundo. La racionalidad dominante del sistema mundo hegemónico descubre la complejidad cuando se confronta con sus límites. La complejidad ambiental irrumpe desde su negación por el pensamiento metafísico y científico, desde la alienación y la incertidumbre del mundo economizado, arrastrado por una racionalidad insustentable y por un proceso incontrolable de producción hacia la muerte entrópica del planeta. (Leff, 2006).

Desde esta perspectiva, la crisis ambiental que nos toca a todos sin ningún distingo y que es además de un problema de conocimiento, un asunto inmediato a nosotros, merece toda atención y constituye una agenda inaplazable en la reflexión de una comunidad de indagación, donde los niños pueden pensar su futuro en un planeta al cual deben cuidar y preservar ya que les ha sido heredado y a su vez lo legarán a futuras generaciones. 
Es inobjetable que asuntos como el biocentrismo, merecen atención inmediata en medio de la crisis ambiental generada por el hombre en medio de la era de la industrialización y el esplendor de la tecnología, que han trascendido el control humano y hacen apuestas al paradigma de desarrollo económico signado por el afán de lucro, cuya racionalidad instrumental socava toda oportunidad de desarrollo sostenible.

El proyecto vital de la Universidad Nacional Abierta y a Distancia (Colombia) apuesta de manera clara por la promoción de comunidades indígenas, campesinas, afrodescendientes, en condiciones especiales, etc., al igual que a miles de colombianos y extranjeros que integran este trazado educativo, pensando en el bienestar y el cultivo del conocimiento disciplinar y transdisciplinar. En esta lógica los grupos de investigación AMAUTAS y GIISCH, circunscriben el observatorio filosófico virtual, para desde su lente enfocar especial interés en el pensamiento filosófico latinoamericano, regional y nacional, considerando baluarte inigualable la prospectiva de las comunidades de indagación y desde allí la generación de ideas encaminadas al cultivo del pensamiento crítico, la creatividad y el asombro, considerando como población particular las niñas y niños de nuestro país.

\section{Referencias}

Alvear, A. E. y Mora, P. A (2011). Observatorio filosófico virtual para el desarrollo de habilidades de pensamiento. Educación y Desarrollo Social. 5(2), 128-140.

https://dialnet.unirioja.es/servlet/articulo?codigo $=5386240$

Amador, B.; Vélez, J.; Cardozo, J.; Pinto, D. y Mora, P. (2017). Argumentación y desarrollo del pensamiento crítico en entornos virtuales de aprendizaje. Colombia: Recuperado de: http://hdl.handle.net/10596/11968

Argüello, A.; Cabeza, O.; Cardona, R.; Hernández, M. y Rodríguez, D. (2012). Del modelo de desarrollo económico al paradigma de desarrollo Humano. Revista Complutense de Educación. 23(2), 401-425

Bauman, Z. (2005). Los retos de la educación en la modernidad líquida. España: Gedisa. 
Barreda, C.; González, C.E.; Constain, G.E. y Mora, P.A. (2019) Determination of the Appropriation Level in the Collaborative Work, a Challenge in Distance Education Focused on E-learning. En: Agredo-Delgado V., Ruiz P. (eds) Human-Computer Interaction. HCl-COLLAB 2018. Communications in Computer and Information Science. 847. Springer.

Boisvert, J. (2004). La formación del pensamiento crítico. Teoría y práctica. México: Fondo de cultura económica.

Derrida, J. (2002). La Universidad sin condición. España: Trotta.

Freire, P. (2002). Concientización: Teoría y práctica de una educación liberadora. Galerna.

Gadotti, M. (2008). Historia de las ideas pedagógicas. México: Siglo XXI Editores.

Giroux, H. A. (2003). Pedagogía y política de la esperanza. Buenos Aires: Amorrortu.

Leff, E. (2006). Aventuras de la Epistemología Ambiental: de la Articulación de las Ciencias al Diálogo de Saberes. México: Siglo XXI Editores.

Morín, E. (2001). Los siete saberes necesarios para la educación del futuro. Francia: UNESCO. Ed. Magisterio.

Nussbaum, M. (2010). Sin fines de lucro, por qué la Democracia necesita de las humanidades. Argentina: Katz Editores.

Paul, R., \& Elder, L. (2003). La mini-guía para el pensamiento crítico, conceptos y herramientas. Dillon Beach, California: Fundación para el pensamiento crítico.

Rodríguez, D.A., Gutiérrez, P., García, S.E., Martínez, N.J., Rincón, E.A. (2019). Correlación de los modelos pedagógicos y el currículo en el contexto educativo. Revista TEMAS, Universidad santo Tomás. III(13), 141-153.

Rodríguez, D. (2019). Influencia de las humanidades en la construcción holística de la ciudadanía universal desde Martha Nussbaum. Bucaramanga, Colombia: Ediciones USTA. Universidad Santo Tomás.

Zuleta, E. (2005). Elogio de la dificultad y otros ensayos. Colombia: Hombre Nuevo Editores. 